\title{
No prevalence of right-left over top-bottom spatial codes
}

\author{
BERNHARD HOMMEL \\ Max Planck Institute for Psychological Research, München, Germany
}

\begin{abstract}
Reaction time is known to depend on spatial stimulus-response compatibility in both the horizontal and the vertical dimensions. However, if both dimensions are varied in the same task, horizontal but not vertical compatibility affects performance, even if subjects are instructed to attend to the vertical dimension only (Nicoletti \& Umiltà, 1984). Experiment 1 compared the effect of horizontal and vertical instructions in a task with left- versus right-handed key responses placed at a top versus bottom location. A horizontal-prevalence effect was observed only with a horizontal, and not with a vertical, instruction. This suggests that subjects might not have heeded the vertical instruction in the Nicoletti and Umiltà study but instead attended to the horizontal dimension. Experiment 2 did not yield any horizontal prevalence with one-handed responses (joystick movements). This indicates that top-bottom codes become ineffective only if the responses suggest an exclusively horizontal response coding. In sum, results demonstrated multiple spatial coding of stimuli and responses under appropriate conditions and suggest that the right-left codes do not dominate the topbottom spatial codes.
\end{abstract}

The stimulus-response (S-R) compatibility phenomenon refers to situations in which particular mapping of a stimulus to a response determines the speed and efficiency of responding. One of the most robust findings in compatibility research is that spatially defined responses are more compatible with spatially corresponding than with noncorresponding stimuli. For instance, if subjects respond to right- and left-side stimuli by pressing a rightand left-hand key, an ipsilateral S-R mapping (i.e., right response to right stimulus and left response to left stimulus) permits faster responses than a contralateral mapping (see, e.g., Brebner, Shephard, \& Cairney, 1972; Nystrom \& Grant, 1955). Likewise, when subjects respond to top and bottom stimuli by pressing an upper and a lower key, performance is better if stimulus and response spatially correspond than if not (e.g., Ladavas \& Moscovitch, 1984; Nicoletti \& Umiltà, 1984). That is, effects of spatial S-R compatibility occur in the horizontal and in the vertical dimension.

However, it has been repeatedly demonstrated by Nicoletti and Umiltà (1984, 1985; Nicoletti, Umiltà, Tressoldi, \& Marzi, 1988) that vertical compatibility ceases to play a role in the presence of horizontal compatibility relations. In Nicoletti and Umiltà's experiments, both horizontal and vertical S-R compatibility were varied orthogonally, so that a particular response

I wish to thank F. Banci and K.-H. Honsberg for constructing the stimulus presentation and response devices; A. Kramer, R. Proctor, and an anonymous reviewer for helpful comments and suggestions; and H. John for checking and improving the English. Correspondence should be addressed to B. Hommel, Max Planck Institute for Psychological Research, Leopoldstr. 24, D-80802 München, Germany (e-mail: hommel@mpipf-muenchen.mpg.de). was horizontally and/or vertically compatible or incompatible to the stimulus. For instance, a top key on the left and a bottom key on the right were pressed in response to a top-left and a bottom-right stimulus, respectively (yielding both horizontal and vertical compatibility), or to a top-right and a bottom-left stimulus (yielding horizontal and vertical incompatibility). Horizontally compatible $S-R$ pairings yielded faster responses than did horizontally incompatible pairings, but there was only a marginal effect of vertical S-R compatibility. This is all the more surprising as the instructions in these experiments referred exclusively to the vertical dimension.

Nicoletti and Umiltà have called this result pattern right-left prevalence and they have already considered, empirically tested, and rejected several explanations. Eventually, Nicoletti et al. (1988) came up with a reference hypothesis. It holds that, although the body midline can serve as a reference for right-left coding, no natural reference axis exists for coding top and bottom. Therefore, horizontal position should be more salient than vertical position and, if both horizontal and vertical cues are available, should dominate vertical position cues.

Unfortunately, this hypothesis has several drawbacks. First of all, it is post hoc and completely arbitrary. It is difficult to see what distinguishes the body midline from any other reference point or axis, including vertical ones. Of course, the body midline differs from other axes in that it divides the body symmetrically, but this provides more problems than advantages for spatial coding (Corballis \& Beale, 1976). Regarding vertical coding, for instance, why shouldn't eye level serve as a reference axis for coding visual stimuli? Ear level might likewise enable the coding of auditory stimuli, and environmental cues might aid spatial coding, too. Moreover, it is not 
clear how the reference hypothesis accounts for pure vertical compatibility effects. If there is no axis for vertical coding, how could top and bottom events ever be discriminated? So, it is fair to say that the reference hypothesis does not provide a satisfactory account for the evidence available.

The possible theoretical relevance of the prevalence effect arises from the fact that it suggests a certain kind of capacity limitation in S-R translation and thus seems to contradict automatic-activation models of S-R compatibility (De Jong, Liang, \& Lauber, 1994; Eimer, Hommel, \& Prinz, in press; Kornblum, Hasbroucq, \& Osman, 1990; Prinz, 1990). According to these models, stimulus codes are formed rather nonselectively and activate codes of compatible (i.e., similar) responses automatically - that is, independently of controlled S-R translation processes. Therefore, some effect of both horizontal and vertical stimulus position on the two-dimensional responses in Nicoletti and Umiltà's (1984, 1985; Nicoletti et al., 1988) studies were expected. As only one dimension yielded an effect, their findings might indicate that automatic-activation models are basically incorrect. In fact, Hasbroucq and Guiard (1991) have argued that there is no need to assume automatic access of stimulus information to response-selection stages besides controlled $\mathrm{S}-\mathrm{R}$ translation. In the same vein, Umiltà and Nicoletti (1990, p. 105) interpreted their right-left prevalence effect as showing "that a subject, if engaged in processing one spatial dimension in a controlled way, is unable to process simultaneously another spatial dimension, not even automatically." From this perspective, the prevalence effect may be due to the fact that, if a response is redundantly signaled by two (spatial) stimulus features, only one is (and can be) selected for controlled S-R translation and thus can affect response speed. Of course, this would not account for why in this case the horizontal but not the vertical code was selected, but we will discuss the reason for that later.

The present studies investigated whether this conclusion is necessary and justified, or whether there is an alternative interpretation. One such interpretation has to do with the instruction that Nicoletti, Umiltà, and colleagues (1984, 1985; Nicoletti et al., 1988) gave to their subjects. In the relevant experiments, stimuli and responses were described exclusively in terms of top and bottom, while terms of left and right were avoided. This, of course, is crucial because with a horizontal instruction, the prevalence of right-left codes would be much less surprising. However, can we really be sure that the subjects followed the instructions and preferred vertical to horizontal stimulus codes?

A comparison of the mean reaction times (RTs) in the experiments of Nicoletti and Umiltà (Table 1) suggests that this was not so. In these experiments, stimuli and responses varied on one dimension only (horizontal or vertical), or on both dimensions. As there always were two stimulus (and response) alternatives in a given subtask (i.e., top-left vs. bottom-right, or top-right vs. bottom-
Table 1

Overview of the Overall Mean Reaction Times (in Milliseconds) in the Experiments of Nicoletti and

Umiltà $(1984,1985)$ and Nicoletti et al. (1988) as a Function of Which Dimensions Were Varied

\begin{tabular}{lccc}
\hline & \multicolumn{2}{c}{ Dimension Varied } & \\
\cline { 2 - 3 } & Horizontal & Vertical & Overall Mean \\
\hline Nicoletti \& Umiltà (1984) & & & \\
$\quad$ Experiment 1 & - & + & 410 \\
Experiment 2 & + & + & 358 \\
Experiment 3 & + & - & 345 \\
Experiment 4 & + & + & 341 \\
Nicoletti \& Umiltà (1985) & & & \\
Experiment 1 & + & + & 316 \\
Experiment 2 & - & + & 367 \\
Experiment 3 & + & + & 320 \\
Nicoletti et al. (1988) & & & \\
Experiment 1 & + & - & 349 \\
Experiment 2 & - & + & 483 \\
Experiment 3 & + & + & 328 \\
\hline
\end{tabular}

left), two-dimensional variations provided redundant information, so that the correct response could be selected on the basis of the information from either dimension. If the subjects had closely followed the instruction, the RT levels of two-dimensional experiments should have been comparable to those of vertical-only experiments. Instead, they resembled those in the horizontal-only experiments, suggesting that the subjects did basically the same under these two conditions.

So, Nicoletti and Umiltà's (1984, 1985; Nicoletti et al., 1988 ) subjects may have simply ignored the instruction, basing their responses on horizontal stimulus codes instead. This makes sense from an economics point of view: As horizontal codes were available about 49$145 \mathrm{msec}$ earlier than vertical codes (see Table 1, singledimension experiments), ignoring the instruction would have permitted much faster responding than would following it. Moreover, if subjects really used (fast) horizontal but not (slow) vertical codes, there would have been no way for vertical codes to affect response selection because responses were already (or about to be) selected at the time of their formation. That is, the prevalence effect need not indicate that vertical codes are not formed at all, but only that they are formed too late to influence response selection.

A second, not necessarily mutually exclusive, interpretation of the prevalence effect concerns the particular responses used in the original studies. Subjects mostly responded with the thumbs of their right and left hands, which, in vertical conditions, were placed on cylinders of differing height. That is, subjects had to spatially discriminate between their hands in order to give a response, so that considering the horizontal dimension was unavoidable for performing the task. If this precluded the coding of responses on the vertical dimension or simply made it superfluous, the ineffectivity of vertical stimulus codes would be hardly surprising: Without a matching response code, the code of a stimulus feature cannot be ex- 
pected to be response compatible or incompatible. So, the prevalence effect may have been a consequence of using dimensionally biased responses.

A similar hypothesis has been considered but then rejected by Nicoletti and Umiltà (1985). In their experiment, subjects responded with the right (or left) hand and the left (or right) foot. That is, an upper and a lower effector were used in a given condition, which was assumed to induce a response coding in terms of top and bottom rather than right and left. As the prevalence effect was present even with this manipulation, the authors rejected the effector-related hypothesis. However, even though one hand was replaced by the corresponding foot in this study, subjects still used a right and a left effector for responding. Although a right-left discrimination between the two hands and between the two feet is an everyday requirement - in deciding which one to useupper and lower limbs will rarely enter into a decision conflict as equivalent response alternatives. This suggests that even when a foot response is opposed with a hand response, hand and foot responses will still be coded in terms of right and left.

The present Experiment 1 was an attempt to replicate the prevalence effect by using the same kind of task as Nicoletti and Umiltà (1984). As in the original study, a vertical instruction was given and right-left terms were avoided completely. However, in order to check whether stimulus-response coding was affected by this instruction at all, a horizontal instruction was given to a second group that carried out an otherwise identical task. If instructions are ignored, the behavior of both groups should be the same; that is, the same compatibility effect(s) should be obtained. In contrast, if instructions are effective, at least the size of compatibility effect(s) should differ between the two groups. So, even if horizontal effects are present and vertical effects absent in either group, horizontal effects should be larger with horizontal than with vertical instruction.

Experiment 2 tested the role of dimensional response bias; subjects performed by moving a joystick with one hand per session only, so that a discrimination between the right and left hands was not required. If the prevalence effect were really due, at least in part, to dimensionally biased responses, then it should not occur with un- biased, that is, one-handed, responses. That is, substantial S-R compatibility effects for both the horizontal and the vertical dimensions were expected in Experiment 2.

\section{EXPERIMENT 1}

The first experiment copied the basic horizontalvertical compatibility task introduced by Nicoletti and Umiltà (1984, Experiment 4). Subjects performed binary choice tasks by pressing microswitches placed diagonally (e.g., top-left vs. bottom-right) in response to stimuli that also accupied the two ends of a diagonal (e.g., topright and bottom-left). Every subject worked through eight subtasks, corresponding to the possible combinations of two stimulus diagonals, two response diagonals, and two $S-R$ mappings. Table 2 shows the resulting eight subtasks. In each subtask, response locations could be spatially compatible or incompatible with the horizontal or the vertical stimulus dimensions, or both. As two subtasks yield the same compatibility relation, respectively, the eight subtasks can be combined to form four compatibility conditions according to the coding scheme in Table 2 (see the two rightmost columns).

In each subtask, stimulus as well as response alternatives differed in both horizontal and vertical location, so that stimuli and responses could be unequivocally described with reference to either dimension. This feature was important for, and was used in, manipulating instruction. In Experiment $1 \mathrm{~A}$, one group of subjects received an instruction that exclusively referred to horizontal stimulus and response locations, while another group received a purely vertical instruction. According to Nicoletti and Umiltà, instruction should not be critical for a prevalence effect to occur at all, so that prevalence of right-left over top-bottom codes-hence, a vertical, but not a (substantial) horizontal, compatibility effect-would be expected in both groups. However, instruction should have a moderating effect, leading to a larger prevalence effect with horizontal than with vertical instruction.

In Experiment $1 \mathrm{~A}$, the response keys were located symmetrically in relation to the subject's eye level in either dimension. In contrast, response keys in the original studies (see, e.g., Nicoletti \& Umiltà, 1984) were both located below eye level. As this methodical difference

Table 2

Overview of Stimuli, Responses, Stimulus-Response Mappings, and the Resulting Compatibility Relation in the Eight Subtasks

\begin{tabular}{cllllll}
\hline Subtask & Stimulus 1 & Response 1 & Stimulus 2 & Response 2 & Horizontal & Vertical \\
\hline 1 & top-left & top-left & bottom-right & bottom-right & + & + \\
2 & top-right & $\begin{array}{l}\text { top-left } \\
\text { bottom-left }\end{array}$ & bottom-right & - & + \\
3 & bottom-left & top-left & $\begin{array}{l}\text { top-right } \\
\text { bottom-right }\end{array}$ & + & - \\
4 & bottom-right & top-left & top-left & bottom-right & - & - \\
5 & top-right & top-right & bottom-left & bottom-left & + & + \\
6 & top-left & top-right & bottom-right & bottom-left & - & + \\
7 & bottom-right & top-right & top-left & bottom-left & + & - \\
8 & bottom-left & top-right & top-right & botiom-left & - & - \\
\hline
\end{tabular}


could be responsible for possible differences in the results, Experiment $1 \mathrm{~B}$ was conducted to test its relevance. The task was the same as in the vertical-instruction group of Experiment 1 A, except that the two response locations were moved downward so that the upper response key was aligned with the subject's eye level.

\section{Method}

Subjects. Twenty-four adult volunteers ( 17 women, 7 men, between 20 and 40 years old) served as paid subjects in single sessions of about $45 \mathrm{~min}$. They reported having normal or correctedto-normal vision and were naive as to the purpose of the experiment. Eight subjects were assigned randomly to either instruction group in Experiment $1 \mathrm{~A}$, and 8 subjects took part in Experiment $1 \mathrm{~B}$.

Apparatus and Stimuli. The experiment took place in a dimly lit room. Stimulus presentation and response measurement were controlled by a Hewlett-Packard Vectra QS20 computer, interfaced with a Data Translation 2821 card for analog input and output. Stimuli were light-emitting diodes (LEDs) mounted on vertical steel rods $82 \mathrm{~cm}$ in front of the subject's chinrest. A central green LED served as fixation point and four red LEDs formed an imaginary square around the fixation light with an edge-to-edge distance of $56 \mathrm{~cm}$ or $36^{\circ}$. The whole stimulus array was covered with fabric, so that unlit LEDs were invisible. Movable horizontal handgrips were mounted on the outside of two vertical metal rods that were located $17 \mathrm{~cm}$ to the right and left of the chinrest. ' Tiny microswitches were taped on the front of the junction piece between grip and rod, so that they could be pressed with the horizontally oriented thumb of the hand holding the grip. For top and bottom responses in Experiment 1A, the grip was moved to a location $22.5 \mathrm{~cm}$ above or below the subject's eye level, respectively. In Experiment IB, the top grip was aligned with the subject's eye level and the bottom grip was located $45 \mathrm{~cm}$ below.

Design and Procedure. The two groups in Experiment $1 \mathrm{~A}$ differed only as to the instructions. These described stimuli and responses exclusively in terms of left and right (horizontal instruction) or in terms of top and bottom (vertical instruction), and subjects were urged to use only the horizontal (or vertical) dimension for stimulus and response coding, respectively. To refer to a particular position, numbers from 1 to 4 were used, with 1 and 2 standing for the top-left and top-right positions, respectively, and 3 and 4 standing for bottom-left and bottom-right positions. In Experiment $1 \mathrm{~B}$, all subjects received a vertical instruction.

Subjects worked through four S-R compatibility conditions (S and $R$ fully compatible, vertically or horizontally compatible, or fully incompatible), each with two subtasks (Table 2). Every subtask consisted of two stimulus and two response alternatives: The two stimulus positions were always located on opposite corners of the imaginary square, such as top left and bottom right. The same was true for response locations, so that a movement to the top-right corner would be paired with a movement to the bottom-left corner. The order of compatibility conditions was balanced across subjects according to a Latin square design. The order of the two subtasks within each compatibility condition was fixed so that the two response positions always alternated between subtasks. In each group, half of the subjects started with the top-left/bottom-right response position and ended with the bottom-left/top-right position; the opposite was true for the other half of the subjects.

After the experimenter had introduced a subtask, the subject could practice as long as he/she desired. Then there were 10 more randomly collected practice trials and 40 experimental trials with 20 replications of each of the two possible S-Rs. Trial order was random, except that the same $S-R$ did not appear more than three times in a row. The sequence of events in each trial was as follows: After an intertrial interval of $2,000 \mathrm{msec}$, the fixation light went on for $500 \mathrm{msec}$, followed by a blank interval of $250 \mathrm{msec}$. Then the stimulus light flashed for $100 \mathrm{msec}$ and the program waited $1,000 \mathrm{msec}$ for a response. Responses with the wrong key were counted as errors and those with latencies exceeding $1,000 \mathrm{msec}$ were considered missing. Latencies below $120 \mathrm{msec}$ were rated as reflecting anticipations. In all of these cases, the trial was recorded and then repeated at some other random position.

\section{Results}

Missing trials $(2.6 \%$ and $2.2 \%$, in Experiments $1 \mathrm{~A}$ and $1 \mathrm{~B}$, respectively; mostly due to the small size of the microswitches), decision errors (1.4\% and $2.5 \%)$, and anticipations $(0.04 \%$ and zero) were not analyzed. From the remaining data, mean RTs were calculated for every combination of horizontal and vertical compatibility, following the coding schema in Table 2 (see Table 3 for means).

Experiment 1A. The data were subjected to a threeway analysis of variance (ANOVA) with horizontal and vertical compatibility as within-subjects variables and instruction as the between-subjects variable. All effects were significant or nearly so: Responses were faster to horizontally compatible than incompatible stimuli $[F(1,14)=32.94, p<.001]$, to vertically compatible than to incompatible stimuli $[F(1,14)=20.30, p<.001]$, and with vertical than horizontal instruction $[F(1,14)=$ $4.28, p<.06]$.

Importantly, both compatibility effects were modified by instruction: Horizontal compatibility effects were larger with horizontal than with vertical instruction (67 vs. $25 \mathrm{msec}$, respectively) $[F(1,14)=6.74, p<.05]$, and vertical compatibility effects were larger with vertical than with horizontal instruction $(42 \mathrm{vs.} 7 \mathrm{msec})[F(1,14)=$ $10.57, p<.01]$. In planned comparisons, all compatibility effects but the vertical under horizontal instruction were significant (at least $p<.05$, one-tailed). Correspondingly, the vertical effect was significantly smaller than the horizontal effect with horizontal instruction, and both effects did not differ under vertical instruction $(p<$

Table 3

Mean Reaction Times (in Milliseconds) in Experiments $1 \mathrm{~A}$ and $1 B$ as a Function of Vertical Compatibility, Horizontal Compatibility, and Instruction

\begin{tabular}{|c|c|c|c|c|}
\hline \multirow{2}{*}{\multicolumn{2}{|c|}{ Vertical Compatibility }} & \multicolumn{3}{|c|}{ Horizontal Compatibility } \\
\hline & & $\mathrm{C}$ & I & {$[-\mathrm{C}$} \\
\hline \multicolumn{5}{|c|}{ Experiment 1A: Horizontal Instruction } \\
\hline $\mathrm{C}$ & & 386 & 451 & 65 \\
\hline I & & 391 & 460 & 69 \\
\hline$I-C$ & $\mathrm{C}$ & 5 & 9 & \\
\hline \multicolumn{5}{|c|}{ Experiment 1A: Vertical Instruction } \\
\hline $\mathrm{C}$ & & 318 & 381 & 63 \\
\hline I & & 398 & 386 & -13 \\
\hline $\mathrm{I}-\mathrm{C}$ & C & 80 & 4 & \\
\hline \multicolumn{5}{|c|}{ Experiment $1 \mathrm{~B}$ : Vertical Instruction } \\
\hline $\mathrm{C}$ & & 320 & 361 & 41 \\
\hline I & & 384 & 385 & 1 \\
\hline $\mathrm{I}-\mathrm{C}$ & $\mathrm{C}$ & 64 & 25 & \\
\hline
\end{tabular}

Note - C, compatible; $\mathrm{I}$, incompatible; I-C, effect size. 
.005 and $p>.28$, respectively). Thus prevalence of horizontal over vertical codes was observed with horizontal instruction only, whereas vertical instruction led to an at least numerical vertical prevalence.

There were two further effects: an interaction of horizontal and vertical compatibility $[F(1,14)=5.39, p<$ $.05]$, which was modified by a three-way interaction involving instruction $[F(1,14)=6.66, p<.05]$. The basis of these effects is obvious (see Table 3 ). Horizontal and vertical compatibility effects combined more or less additively to the RT pattern with horizontal instruction, but vertical instruction yielded an underadditive pattern. So, with horizontal incompatibility, vertical incompatibility led to faster responses than with vertical compatibility.

Experiment 1B. A two-way ANOVA with horizontal and vertical compatibility as within-subjects variables produced a highly significant effect of vertical compatibility $[F(1,7)=30.67, p<.001]$, whereas the horizontal effect and the interaction only approached significance $(.05<p<.10)$. That is, there was no sign of a prevalence of horizontal over vertical codes, but rather, the opposite.

To test the effect of absolute response location (centered at vs. below eye level), an additional three-way ANOVA was run over the data from the verticalinstruction groups of Experiments 1A and 1B. The main effects of horizontal compatibility $[F(1,14)=13.99, p<$ $.005]$ and of vertical compatibility $[F(1,14)=53.38, p<$ $.001]$ were significant, as was the interaction between them $[F(1,14)=14.75, p<.005]$. However, absolute response position (i.e., the experiment factor) did not participate in or produce any effect $(p s>.24)$.

\section{Discussion}

Although the designs of Experiments $1 \mathrm{~A}$ and 1B closely followed those of the original experiment of Nicoletti and Umiltà (1984, Experiment 4), results did not replicate. First, there was no indication of any prevalence of left-right over top-bottom codes with vertical instruction (as used by Nicoletti and Umiltà). Instead, vertical compatibility effects were larger than horizontal effects, which, in view of the instruction, is not too surprising. This was true irrespective of whether responses were centered at, or situated below, the subject's eye level, as in the original studies, precluding the possibility that absolute response location was responsible for the failure to replicate. It seems that subjects were able to attend to the emphasized stimulus (or response) dimension, thus producing a stronger effect of compatibility relations defined on this dimension. This rules out Nicoletti et al.'s (1988) assumption that the horizontal dimension is per se more salient than the vertical and questions the generality of Nicoletti and Umiltà's (1984, 1985; Nicoletti et al., 1988) findings.

Second, the two compatibility effects interact or at least tend to do so under vertical instruction. This is another demonstration that both spatial stimulus dimensions were coded and thus affected RT, even though one of them was completely uninformative. So, there is little evidence for the claim that only one code is formed with redundant dimensions. Although the underadditive pattern of the dimensional interaction might seem counterintuitive at first sight, it makes sense from an acting subject's point of view. Of course, complete compatibility provides the easiest condition, because the relative response location is directly given by the stimulus. With complete incompatibility, however, subjects often follow the rule to do the opposite of what the stimulus suggeststhat is, use stimulus location as a starting point for an "away" response. No such simple rule can be established with mixed compatibility relations (provided that both stimulus dimensions are coded), which might account for the underadditive result pattern. The theoretical implications of this observation will be addressed in the General Discussion.

Third, however, although no evidence of a prevalence of horizontal over vertical stimulus codes was found in the vertical-instruction condition of Experiment $1 \mathrm{~A}$ or in Experiment 1B, a comparison between the horizontaland the vertical-instruction conditions of Experiment $1 \mathrm{~A}$ does reveal an asymmetry between stimulus dimensions. Under both conditions, compatibility effects were larger for the dimension emphasized in the instruction, showing that instruction had an effect on spatial stimulus (and/or response) coding. However, whereas vertical instruction allowed for a substantial effect of horizontal compatibility, there was virtually no vertical compatibility effect with horizontal instruction. So, there is indeed a kind of prevalence of horizontal over vertical codes, although only under specific conditions.

The present results suggest that subjects in the Nicoletti and Umiltà (1984, 1985; Nicoletti et al., 1988) studies did not follow the vertical instructions but instead attended to the horizontal dimension. If so, Nicoletti and Umilta's findings must be compared with those for the horizontal, but not the vertical, group of Experiment 1A, and this comparison turns out well: In both cases, there is little evidence for an effect of vertical compatibility. Interestingly, the present findings rule out that this was due to trivial temporal reasons. In Nicoletti and Umiltà's studies, vertical code formation took at least $50 \mathrm{msec}$ longer than the formation of horizontal codes, so that vertical codes may have often been formed simply too late to affect response selection. In the present experiments, however, vertical instruction allowed for faster responses than did horizontal instruction, showing that a purely temporal explanation for the disappearance of vertical effects does not suffice. So, another account is needed, probably in terms of dimensionally biased responses. Experiment 2 was conducted to substantiate this idea.

\section{EXPERIMENT 2}

The main outcome of Experiment 1 was that the salience of the horizontal and the vertical dimension did 
not seem to be as differently pronounced as Nicoletti et al. (1988) assumed. Only with horizontal instruction was there an indication that vertical codes may be dominated by horizontal codes. A possible reason for this asymmetry might be that the left and right hands were used for responding. Responses that are usually discriminated by referring to the horizontal dimension may suggest or require horizontal response coding, whereas vertical codes are only formed optionally. As a consequence, horizontal response codes would be formed (and thus can be activated by matching stimulus codes) under any instruction, but vertical codes would be formed under vertical instruction only.

An obvious means to test this response-bias hypothesis would be to avoid using left-versus right-hand responses. So, in this experiment, subjects used only one hand per session for moving the lever of a joystick to the top-left, top-right, bottom-left, or bottom-right position. In other respects, the task was the same as in Experiment $1 \mathrm{~A}$, including the two-instruction manipulation. According to the response-bias hypothesis, horizontal and vertical compatibility effects should be present under either instruction, even though their relative strength might vary with instruction type.

\section{Method}

Subjects. Twenty-four adult volunteers ( 9 women, 15 men, between 19 and 40 years old) served as paid subjects in two sessions of about $45 \mathrm{~min}$ each. They reported having normal or correctedto-normal vision and were naive as to the purpose of the experiment. Twelve subjects were assigned randomly to each instruction group.

Apparatus and Stimuli. These were as in Experiment 1 except for the response device. A modified analog precision joystick from a model airplane control device was installed $25 \mathrm{~cm}$ in front of the subject, with an angle of $55^{\circ}$ to the horizontal plane, so that the stick's top end was located about $10 \mathrm{~cm}$ beneath the subject's chin. Subjects responded by moving the hand-held joystick lever diagonally to the upper or lower right or left, depending on the stimulus and the S-R mapping, which corresponded to a deflection from zero to a position of about $40^{\circ}$. Hand and arm postures were somewhat constrained by the two vertical metal rods used in Experiment 1 , which had to be embraced to hold the joystick.

Design and Procedure. These were largely identical to those in Experiment 1 . Subjects used their left hands in one session and their right hands in the other, in counterbalanced order. During a session, they worked through the same four S-R compatibility conditions (or eight subtasks) as those used in Experiment 1 (see Table 2). The 24 (4!) possible orders of compatibility conditions were randomly assigned to the 24 sessions ( 12 subjects $\times 2$ sessions) of each instruction group, and the order of the two subtasks within each compatibility condition was also balanced within groups across subjects and sessions.

\section{Results}

There were no anticipations. Missing trials (1.4\%, mostly due to aiming errors or technical problems) were as seldom as decision errors $(1.6 \%)$ and were not analyzed. The remaining data were treated as in Experiment 1 (see Table 4 for means).

As in Experiment 1, responses were faster to horizontally compatible than incompatible stimuli $[F(1,22)=$
Table 4

Mean Reaction Times (in Milliseconds) in Experiment 2 as a Function of Vertical Compatibility, Horizontal Compatibility, and Instruction

\begin{tabular}{cccr}
\hline & \multicolumn{3}{c}{ Horizontal Compatibility } \\
\cline { 2 - 4 } Vertical Compatibility & C & I & I-C \\
\hline & Horizontal Instruction & & \\
C & 421 & 483 & 62 \\
I & 491 & 470 & -20 \\
I-C & 70 & -13 & \\
& Vertical Instruction & & \\
C & 356 & 406 & 50 \\
I & 448 & 417 & -30 \\
I-C & 91 & 11 & \\
\hline
\end{tabular}

Note-C, compatible; I, incompatibility; I-C, effect size.

$9.02, p<.01]$, to vertically compatible than incompatible stimuli $[F(1,22)=49.90, p<.001]$, and with vertical than with horizontal instruction $[F(1,22)=6.35, p<$ .05 ]. Again, the horizontal effect was larger under horizontal than under vertical instruction $(21$ vs. $10 \mathrm{msec}$, respectively) and the vertical effect was larger under vertical than under horizontal instruction $(5 \mathrm{l}$ vs. $28 \mathrm{msec}$, respectively). However, the first interaction was far from significant $(p>29)$ and the second produced only a borderline effect $[F(1,22)=4.07, p<.06]$. Planned comparisons showed that the vertical compatibility effect did not differ from the horizontal effect under horizontal instruction and was even larger than that under vertical instruction ( $p>.4$ and $p<.01$, respectively). As in Experiment 1 , horizontal and vertical compatibility interacted underadditively $[F(1,22)=77.67, p<.001]$, but there was no further modification by instruction $(p>9){ }^{2}$

\section{Discussion}

As predicted by the proposed response-bias hypothesis, there was no indication for any prevalence of rightleft over top-bottom codes in this experiment. On the contrary, both horizontal and vertical compatibility yielded strong effects that also interacted underadditively with one another. So, the results demonstrated that both dimensions can produce substantial compatibility effects in a two-dimensional task even under horizontal instruction. This outcome is inconsistent with the findings of Nicoletti and Umiltà $(1984,1985$; Nicoletti et al., 1988) and supports the assumption that their choice of dimensionally biased responses was responsible for their rightleft prevalence effect. If anything, the present experiment demonstrates a prevalence of top bottom codes: Whereas Nicoletti and Umiltà (1984), who found the largest vertical effect in a two-dimensional task, obtained a horizontal effect of $62 \mathrm{msec}$ and a vertical effect of only $17 \mathrm{msec}$, the present experiment produced a completely reversed data pattern, namely $15 \mathrm{msec}$ and $40 \mathrm{msec}$. However, this difference in magnitude between horizontal and vertical effects is of little significance in view of the pronounced cross-over interaction that was obtained. 


\section{GENERAL DISCUSSION}

The purpose of the present study was to test two possible, nonexclusive accounts of the prevalence of rightleft over top-bottom spatial codes repeatedly found by Nicoletti and Umiltà (1984, 1985; Nicoletti et al., 1988). The first account concerned the instruction. There is evidence that Nicoletti and Umiltà's subjects did not follow the vertical instruction given to them, but rather, the horizontal instruction instead. In Experiment 1A, instruction was varied and was in fact involved as a factor in several interaction effects. That is, instruction was effective in this experiment. However, the results showed that vertical instruction did not produce any right-left prevalence, which is clearly inconsistent with both Nicoletti and Umiltà's findings and their claim that the horizontal dimension per se is more salient than the vertical one. With horizontal instruction, a result pattern quite similar to that of Nicoletti and Umiltà's studies was observed. This supports our reasoning that the original prevalence effect was due to the fact that the subjects did not follow the instruction.

What remained to be explained was why a prevalence effect occurred under horizontal instruction at all. Experiment 2 tested a response-bias account that attributes this effect to the concurrent use of both hands in the same task. As predicted, even the restricted prevalence effect found in Experiment 1A disappeared with onehanded responses. These findings are not consistent with the idea that there may be something special to right and left positions or codes, and, thus, rule out the reference hypothesis of Nicoletti et al. (1988). Whatever reference system our subjects used for locating top and bottom stimuli and responses, there must be one that allows for some coding that is as efficient as the body midline (or an equivalent) is for horizontal coding. Experiment $1 \mathrm{~B}$ showed that it does not matter whether the responses are placed at or below eye level, which suggests that the reference system used for vertical coding does not originate from, and is not centered at, eye or head level. Whatever the prevalence effect is, we can conclude that, if present at all, it does not reflect some basic asymmetry between horizontal and vertical stimulus or response codes, but is produced by the choosing of responses that are usually or preferredly coded as left and right.

In the present context, it is important not to confuse spatial response or effector codes with the anatomical identity of the effectors. It is well known that what matters in spatial S-R compatibility effects is not the correspondence between stimulus and anatomical hand, but that between stimulus and hand position (see, e.g., Wallace, 1971) or action goal position (Guiard, 1983; Hommel, 1993). Consistent with that, the anatomical identity of the hand had virtually no effect on S-R compatibility in Experiment 2 (apart from the opaque higher order interaction mentioned in note 2). Judging from this, the difference between the outcomes of Experiments 1 and 2 is not likely to be related to anatomical factors, but rather to the discrimination between, and the coding of, relative positions of concurrently used effectors. In line with that, the response-bias hypothesis refers to spatial codes of effectors but not to their anatomical identity. That is, its predictions do not depend on which hand operates which key, so that the same results would have been expected in Experiment $1 \mathrm{~A}$ if the subjects had had their hands crossed.

Although the importance of spatial response coding is well established, little is known about the rules it follows. The only available evidence comes from singular observations - for example, that right-left response coding and thus compatibility effects are more pronounced in volleyball players than in soccer players, presumably because volleyball is characterized by a stronger functional specialization of right versus left effectors than is soccer (Castiello \& Umiltà, 1987). If this means that right versus left response coding is the more pronounced the more important the spatial discrimination of the effectors in question is, it would fit well with the present findings. However, it is clear that a principled account of response coding is lacking and, as long as this is so, theories on S-R compatibility remain necessarily incomplete (Hommel, 1993).

An unexpected outcome of the present study is the finding that the direction of compatibility effects of one dimension depended on the compatibility of the other dimension. That is, the benefit of spatial S-R correspondence on one dimension decreases, and can even be inverted, if there is incompatibility on the other dimension. Interestingly, interactions of two spatially defined dimensions have been found before (Ehrenstein, 1994; Michaels, 1988; Proctor, Van Zandt, Lu, \& Weeks, 1993), and there is a further obvious parallel to an interaction of spatial and nonspatial dimensions first reported by Hedge and Marsh (1975). Hedge and Marsh's subjects always responded to red and green stimuli by pressing a red and a green response key. One key was located on the right and one on the left side, and the stimuli appeared randomly on the right or left side of the display. Nevertheless, for both stimuli and responses, only color was important, not location. In one condition, the S-R mapping was direct; that is, red stimulus to red key and green stimulus to green key. Similar to what has been found by others (e.g., Simon \& Rudell, 1967), responses were faster with spatial S-R correspondence, hence when the stimulus appeared on the side of the correct response. In another condition, the S-R mapping was reversed; that is, red stimulus mapped to green key and green stimulus to red key. Here, responses were slower with spatial S-R correspondence than with noncorrespondence. So, the reversal of an originally direct $S-R$ relationship between response and relevant stimulus attribute did reverse the effect of (irrelevant) spatial S-R compatibility, too.

A possible account for these cross-over effects has been suggested by Hedge and Marsh (1975) themselves and, in slightly revised versions, by Arend and Wandmacher (1987) and Lu and Proctor (1994). The general 
idea is that, with alternate mappings, the relevant stimulus information undergoes some kind of inversion operation, or logical recoding, in order to form the response code. If this operation affected not only the primary but also the secondary information, the code of the secondary or irrelevant stimulus attribute would be unintentionally transformed into its opposite. Assume that our subjects followed the instructions very closely and thus treated only one of the two dimensions as relevant and the other as irrelevant. With vertical instruction, for instance, vertical stimulus position would then have played the role of the relevant stimulus and horizontal position that of the irrelevant stimulus. With vertical compatibility, the vertical stimulus code would directly specify the correct response, so that no $\mathrm{S}-\mathrm{R}$ translation or transformation would be necessary. Consequently, horizontal codes remain unaltered and would thus prime the spatially corresponding response. With vertical incompatibility, however, the vertical code may undergo a logical recoding operation before the correct response is selected. If so, the horizontal information may be transformed into its opposite, too, so that left stimuli prime right responses and vice versa. The expected outcome would be a cross-over interaction of vertical and horizontal compatibility of the kind actually obtained. So, a logical recoding approach provides a reasonable account of our findings as well as for the similar results of Michaels (1988), Proctor et al. (1993), and Ehrenstein (1994).

An important theoretical implication of the present results is that, obviously, more than one spatial code can be formed for both stimulus and response. Although the occurrence of prevalence effects might have suggested that there is a limitation in the number of codes to one, our findings clearly demonstrate that stimuli as well as responses must have been coded both horizontally and vertically. Also, the obtained interaction between horizontal and vertical compatibility implies that two different codes, one for each dimension, were formed for stimuli and responses at the same time, or at least during the same time interval. Of course, this may have been enforced by the particular task and the instruction, so that other tasks may well enable the formation of unitary spatial codes. However, we can safely conclude that the coexistence of different spatial stimulus codes and different spatial response codes is generally possible.

The demonstration of multiple, interdimensional spatial coding complements findings of intradimensional concurrent coding of horizontal position and destination (Ehrenstein, 1994; Michaels, 1988; Proctor et al., 1993); of absolute and relative horizontal stimulus position (Lamberts, Tavernier, \& d'Ydewalle, 1992; Umiltà \& Liotti, 1987); and of hand location, anatomical mapping of hand, and response goal location (Hommel, 1993). In these studies, most of the spatial stimulus and response attributes were completely irrelevant to the task, so that code formation was neither necessary nor helpful. The same was true for the present experiments: As horizon- tal and vertical positions were perfectly correlated in each condition, our subjects could well afford to completely ignore one of the two spatial dimensions. The fact that they did not do so strongly suggests, as do the intradimensional findings, that the spatial features of stimulus and response events are coded automatically, at least those features about which there is uncertainty.

This outcome contradicts Umiltà and Nicoletti's (1990) claim that attending to one spatial stimulus dimension prevents the formation of codes related to another spatial dimension. In doing so, it rules out a possible objection to the automatic stimulus-coding assumption inherent in automatic-activation models of S-R compatibility (Eimer et al., in press; De Jong et al., 1994; Kornblum et al., 1990; Prinz, 1990). In line with this assumption, the present study demonstrates that although attending to one spatial dimension can increase the impact of this dimension, it does not preclude the processing of another. Although this impedes performance in artificial tasks like ours, with completely arbitrary combinations of stimulus and response locations, automatic spatial coding would seem very useful for everyday action planning and action control, because goal-directed limb and body movements need, first of all, spatial information about action-relevant stimulus events. So, performance in spatial compatibility experiments does not always reflect, but is likely to reveal, the expediency of perceptionaction coordination.

\section{REFERENCES}

AREND, U., \& Wandmacher, J. (1987). On the generality of logical recoding in spatial interference tasks. Acta Psychologica, 65, 193-210.

Brebner, J., Shephard, M., \& Cairney, P. (1972). Spatial relationships and S-R compatibility. Acta Psychologica, 36, 1-15.

Castiello, U., \& Umiltà, C. (1987). Spatial compatibility effects in different sports. International Journal of Sport Psychology, 18, 276-285.

Corballis, M. C., \& Beale, I. L. (1976). The psychology of left and right. Hillsdale, $\mathrm{NJ}$ : Erlbaum.

De Jong, R., Liang, C.-C., \& Lauber, E. (1994). Conditional and unconditional automaticity: A dual-process model of effects of spatial stimulus-response correspondence. Journal of Experimental Psychology: Human Perception \& Performance, 20, 731-750.

Ehrenstein, W. H. (1994). The Simon effect and visual motion. Psychological Research/Psychologische Forschung, 56, 163-169.

Ermer, M., Hommel, B., \& Prinz,W. (in press). S-R compatibility and response selection. Acta Psychologica.

GutaRD, Y. (1983). The lateral coding of rotations: A study of the Simon effect with wheel-rotation responses. Journal of Motor Behavior, 15, 331-342.

HasbroucQ, T., \& Guiard, Y. (1991). Stimulus-response compatibility and the Simon effect: Toward a conceptual clarification. Journal of Experimental Psychology: Human Perception \& Performance, 17, 246-266.

Hedge, A., \& Marsh, N. W. A. (1975). The effect of irrelevant spatial correspondences on two-choice response-time. Acta Psychologica, 39, 427-439.

Hommel, B. (1993). Inverting the Simon effect by intention: Determinants of direction and extent of effects of irrelevant spatial information. Psychological Research/Psychologische Forschung, 55, 270279 .

Kornblum, S., Hasbrouce, T., \& Osman, A. (1990). Dimensional overlap: Cognitive basis for stimulus-response compatibility-A model and taxonomy. Psychological Review, 97, 253-270. 
Ladavas, E., \& Moscovitch, M. (1984). Must egocentric and environmental frames of reference be aligned to produce spatial S-R compatibility effects? Journal of Experimental Psychology: Human Perception \& Performance, 10, 205-2 15.

Lamberts, K., Tavernier, G., \& D'Ydewalle, G. (1992). Effects of multiple reference points in spatial stimulus-response compatibility. Acta Psychologica, 79, 115-130.

Lu, C.-H., \& Proctor, R. W. (1994). Processing of an irrelevant location dimension as a function of the relevant stimulus dimension. Journal of Experimental Psychology: Human Perception \& Performance, 20, 286-298.

Michaels, C. (1988). S-R compatibility between response position and destination of apparent motion: Evidence of the detection of affordances. Journal of Experimental Psychology: Human Perception \& Performance, 14, 231-240.

Nicoletti, R., \& Umiltà, C. (1984). Right-left prevalence in spatial compatibility. Perception \& Psychophysics, 35, 333-343.

NicoletTl, R., \& UmiLtà, C. (1985). Responding with hand and foot: The right/left prevalence in spatial compatibility is still present. Perception \& Psychophysics, 38, 211-216.

Nicoletti, R., Umiltà, C., Tressoldi, E. P., \& Marzi, C. A. (1988). Why are left-right spatial codes easier to form than above-below ones? Perception \& Psychophysics, 43, 287-292.

Nystrom, C. O., \& Grant, D. A. (1955). Performance on a key-pressing task as a function of the angular correspondence between stimulus and response elements. Perceptual \& Motor Skills, 5, 113-125.

PRINZ, W. (1990). A common coding approach to perception and action. In O. Neumann \& W. Prinz (Eds.), Relationships between perception and action (pp. 167-201). Berlin: Springer-Verlag.

Proctor, R. W., Van Zandt, T., Lu, C.-H., \& Weeks, D. J. (1993). Stimulus-response compatibility for moving stimuli: Perception of affordances or directional coding? Journal of Experimental Psychology: Human Perception \& Performance, 19, 81-91.
Simon, J. R., \& Rudel, A. P. (1967). Auditory S-R compatibility: The effect of an irrelevant cue on information processing. Journal of Applied Psychology, 5t, 300-304.

UMiLTÀ, C., \& LIOTTI, M. ( 1987 ). Egocentric and relative spatial codes in S-R compatibility. Psychological Research, 49, 81-90.

Umiltà, C., \& Nicolettı, R. (1990). Spatial stimulus-response compatibility. In R. W. Proctor \& T. G. Reeve (Eds.), Stimulus-response compatibility (pp. 89-116). Amsterdam: Elsevier, North-Holland. WALLACE, R. J. (1971). S-R compatibility and the idea of a response code. Journal of Experimental Psychology, 88, 354-360.

\section{NOTES}

1. For technical reasons, it was not possible to use the same horizontal distance between responses as in the original studies, yielding a somewhat decreased horizontal response discriminability. However, there is no indication that this affected the outcome: Even the most plausible candidate - the disadvantage of horizontal to vertical instruction in Experiment 1 -was replicated in Experiment 2, in which completely different responses were used.

2. Additional, more finely grained analyses made sure that the obtained effects did not depend on-hence, did not interact withresponse hand. The only exception was a hand $x$ horizontal stimulus position $\times$ horizontal response position $\times$ vertical response position interaction under horizontal instruction. This was because, for nonobvious reasons, the horizontal compatibility effect was larger with upward than with downward movements of the left hand $(27 \mathrm{vs} .20 \mathrm{msec}$, respectively), but smailer with upward than with downward movements of the right hand ( $14 \mathrm{vs}$. $23 \mathrm{msec}$, respectively).

(Manuscript received June 14, 1994; revision accepted for publication June 15, 1995.) 